\title{
Magnetic Properties of Nanoparticles
}

\author{
Mashal Alenazi \\ University of Bridgeport, Biomedical Engineering, USA
}

\begin{abstract}
The paper suggests strongly that the tendency and outcome of surface segregation in magnetic alloy nanostructures should be taken into full account in the design, fabrication, and employment of these magnetic nanostructures. the role of macrostrain in altering the magnetization of $\mathrm{BiFeO}$ nanoparticles is not discussed in detail, making it an indispensable study at this juncture. Strain engineering to control physical properties of materials is an emerging area of research. a systematic investigation on the temperature and size-dependent changes in the magnetic properties of Pd NPs is presented by comparing the temperature-dependent magnetic behavior of high-purity bulk Pd with those of NPs of Pd with diameter D = $12 \mathrm{~nm}$ and $7.7 \mathrm{~nm}$. Attention is given to not only how the ferromagnetic component changes with size D but also the maxima at Tm, the later often neglected in the previous studies on Pd NPs.
\end{abstract}

Keywords: Magnetic, Nanoparticles, Microemulsions, Electrodes.

\section{INTRODUCTION}

Nanosized magnetic structures are currently key materials above this value. However, the percolation threshold can for advancements in electronics, optoelectronics, Magnetic have a wide range, from a little as $15 \%$ metal all the way storage, and many bioinspired applications. What is to $80 \%$. One can suppose ohmic or tunnelling conductivity usually termed "nanostructured systems" comprises those in the structures. In the case of ohmic conductivity so materials whose properties are determined by entities called infinite cluster - objects connected to both particles, crystallites, or clusters! with characteristic electrodes applied to opposite sides of the structure - is lengths between 1 and $100 \mathrm{~nm}$ in at least two dimensions. analysed by the help of modified burning method, which If the grain or domain size becomes comparable or smaller takes into account spherical objects with real centre to the characteristic length scale of the interaction coordinates.

processes controlling a particular property, different effects and unusual chemical and physical properties can be expected that are highly attractive in a number of technical applications. Nanoparticles of barium ferrite (BaFe12O19) were synthesized using a novel method called microemulsion processing. In this process, the aqueous cores (typically $5-25 \mathrm{~nm}$ in size) of watercetyltrimethylammonium bromide - n-butanol-octane microemulsions were used as constrained microreactors for the co-precipitation of precursor carbonates (typically $5-15 \mathrm{~nm}$ in size). The carbonates thus formed were separated, dried and calcined to form nanoparticles (less than $100 \mathrm{~nm}$ ) of barium ferrite. Specification of such structures requires topological and geometrical descriptors so we are able to characterise the connectivity and spatial configuration.

In the last years, new advanced techniques have been developed in spatial statistics. Generally, it may be stated that today there is strive for use of multipoint statistic correlation functions for morphology analysis. The electric properties of composite or nanocomposite films come in on foreground of interest, especially near the percolation threshold. The parameter which influences the electric properties of composite structures is the metal volume fraction [1] contained in dielectric material. The metal particles are completely insulated from each other at low values of metal volume fraction, near the critical value of it they form a percolation structure, and the structure embodies the metallic behaviour with dielectric inclusions

Adhesion and diffusion of the drug in the mucosal microenvironment of the GI tract is another problem for oral chemotherapy. Before the drug molecules reach their final destination (e.g., the blood system, the lymphatic system, the target tissue or cell), it must go through the stomach, the lumen of the intestine, the mucus layer coating the intestinal epithelium, and finally the epithelium itself. The human intestinal epithelium is highly absorptive and is made up of the villi that vastly increase the total surface area of the epithelium available for absorption of the drug in the GI tract, which could be as large as approximately $400 \mathrm{~m} 2$. Absorptive enterocyte cells and mucus secreting goblet cells cover the villi, which are interspersed with follicle associated epithelium (FAE). These lymphoid nodules, Peyer's patches, are covered with microfold cells (M cells) specialized for antigen sampling. M cells are significant for drug delivery [29] since they are relatively less protected by mucus and possess a high transcytotic activity.

Magnetic FePt nanoparticles have promising applications in permanent magnets and ultrahigh-density magnetic information storage. Much progress has been accomplished on synthesizing FePt nanoparticles with well-controlled shape size, and overall composition.

However, the magnetic performance of these $\mathrm{FePt}$ nanoparticles was found not as excellent as hoped. It has been widely recognized that the beneficial L1 chemical ordering in the FePt nanoparticles would diminish with 
decreasing particle size, whereas the magnetic properties electron bulk conductivity of the support to efficiently of the FePt nanoparticles depend strongly on such transfer the electrons to the electrode. Over the last years, chemical ordering in these particles. Surface segregation is a variety of supports, such as carbon nanotubes, graphene believed to be one of the main material processes that are , carbon fibers,, and conductive polymers have been tested responsible for the chemical disordering in the FePt in electrocatalytic applications such as fuel cells and nanoparticles. Specifically, the Pt atoms were predicted to biosensors. C60 is a less studied support, as explained by occupy surface sites more preferably than the Fe atoms in the lack of a "one-dimensional" structure and a relatively the FePt nanoparticles [2].

\section{CORE-SHELL MODEL FOR THE MAGNETIC PROPERTIES}

FePt nanoparticles. For the cuboid nanoparticle, the surface segregation caused the MAE decrease from 0.97 to $0.56 \mathrm{meV} / \mathrm{atom}$. More pronouncedly, the surface segregation in the cuboctahedral nanoparticle reduced the MAE from 2.74 to $0.03 \mathrm{meV} / \mathrm{atom}$. It is worth mentioning that we did not find any significant changes caused by the surface segregation in the spin- or orbital- magnetic moment anisotropies (D1S and Dl ) as well as the ratio of orbital and spin moments (lLL/l) of our simulated nanoparticles. As mentioned above, the Fe atoms which move away from their original lattice sites contribute most to the reduction in the magnetic moments of the surfacesegregated FePt nanoparticles. Additionally, we observed that the surface segregation also causes a decrease in the magnetic anisotropy of the surface-segregated FePt nanoparticles. A recent study showed it was the Fe atoms in the Fe sublattice that contribute most to the MAE of the L1 0 FePt crystal. Hence, it is inferred that the Fe atoms which move away from their original lattice sites due to the surface segregation are also responsible for the observed reduction in the MAE of the surface-segregated FePt nanoparticles. The magnetic properties of the FePt nanoparticles are pertinent to their performance in their applications [4].

The reduced dimensionality of the nanoparticles (NPs) and a larger proportion of their atoms on the surface usually lead to size-dependent properties of NPs. This is because surface atoms of NPs experience reduced symmetry compared to atoms in the core of a NP producing unsaturated bonds on surface atoms and associated surface energy and anisotropy [1]. In heterogeneous catalysis, surface adsorption of a gaseous molecule such as $\mathrm{H} 2$ on a catalyst in the atomic state is usually the first step since it is energetically favorable to compensate the unsaturated bonds and lower the surface energy [2]. To increase the proportion of the surface atoms whose number density increases inversely with the size D of the NPs, highly dispersed catalysts with nanoscale dimensions are usually used in catalysis. The different electronic states of surface atoms can also affect the magnetic properties of the NPs in a similar manner providing correlation between catalysis and magnetism [1].

the properties of the support - involving processes such as fuel diffusion (for fuel cell application), good anchoring of catalysts to the support ensuring both long-term stability and efficient electron conductivity, and finally a good low conductivity. However, the low conductivity of C60 originates solely from a low concentration of charge carriers, due to its large bandgap of $1.6 \mathrm{eV}$. In fact, the electron mobility of crystalline $\mathrm{C} 60$ is rather high, making it popular in, for example, solar cell and and field effect transistor (FET) applications.

The mixture was heated under nitrogen atmospheres at 55 C after degassing using six alternating cycles of evacuation and pressurization with high purity nitrogen (starting with evacuation). Polymerization carried out after the injection of a CoBF/MAA mixture (7.5 mg CoBF dissolved in $74.0 \mathrm{~g}$ MAA). The reaction was allowed to proceed for $2 \mathrm{~h}$ with continuous stirring under nitrogen atmosphere and then quenched with an ice-water bath. The as-prepared PMAA was precipitated in diethyl ether, and then redissolved in DI water and reprecipitated in diethyl ether for several times. UV-vis absorption spectra were taken with a Perkin-Elmer Lambda 900 UV-vis spectrometer with the scan rang from 260 to $450 \mathrm{~nm}$ using DMF as solvent. The powder X-ray diffraction (XRD) patterns were conducted on a Bruker-AXS D8 ADVANCE $\mathrm{X}$-ray diffractometer at a scanning rate of $6^{\circ} / \mathrm{min}$ in $2 \theta$ ranging from $10^{\circ}$ to $80^{\circ}$ with $\mathrm{CuK} \alpha$ radiation $(\lambda=0.1542$ $\mathrm{nm}$ ). Fourier transform infrared (FT-IR) spectra were recorded on a NICOLET-NEXUS 670 spectrometer. The samples were grounded with $\mathrm{KBr}$ crystal and the mixture of them was pressed into a flake for IR measurement.

The changes of melting enthalpy, $\mathrm{H}$, of the PLA/MMT system prepared by the melt extrusion were considerably higher than those obtained by solvent dissolution method. It was considered that the binding force between PLA and inorganic compound in the composite prepared by melt extrusion method was higher than in those prepared by solvent dissolution method. The automotive industry makes frequent use of the cast multicomponent hypoeutectic $\mathrm{AlSiCu}$ alloys. These alloys have been characterized by presence of two $\mathrm{Al}-\mathrm{Si}$ and $\mathrm{Al}-\mathrm{Si}-\mathrm{Cu}$ eutectics, which are primarily responsible for defining the microstructure and mechanical properties of these alloys. Comprehensive understanding of solidification paths of these alloys is of paramount importance for metallurgical engineers. This knowledge also enable the process, quality and simulation engineers as well as designers to ensure that the casting will achieve the desired properties for its intended application after corresponding melting, liquid metal processing, mold filling and heat treatment procedure. In order to ensure that cast components have good mechanical properties their as-cast microstructures must be closely monitored [3]. The solidification path of $\mathrm{AlSiCu}$ alloys can be studied using thermal analysis technique. 
Application of the thermal analysis technique in the ferrous and non-ferrous casting industry has been already many years successfully employed. Cooling curve analysis has been used for many years to determine binary phase diagrams and for fundamental metallurgical studies. In using this technique, binary alloys of varying composition were studied as they cooled and the arrest points were recorded and plotted on a temperature - composition (phase) diagram. The cooling curve method is useful for commercial applications for a number of reasons: it is simple, inexpensive and provides consistent results [5].

\section{CONCLUSION}

bulk-like contribution from the atoms in the core of the NPs yielding the presence of Tm in the $\chi$ vs. T data and ferromagnetic component which increases with decrease in particle size and so it is due to the atoms on the surface of the NPs. The fact that this ferromagnetism can be modified with different capping agents confirms this coreshell model of the magnetism of Pd NPs. Change in ferromagnetism with different capping agents likely occurs as a result of charge (electron) transfer between the capping agent and surface atoms, as shown convincingly for capped $\mathrm{CdSe}$ nanoparticles. The enhancement of bioavailability of poorly water-soluble drugs is one of the most challenging problems in pharmaceutical science. Drug-polymer solid dispersion can markedly improve the dissolution rate of drugs and lead to higher bioavailability (Chiou and Riegelman, 1971, Leuner and Dressman, 2000 and $\mathrm{Yu}, 2001)$. However, due to the lack of miscibility between most of the insoluble drugs and commonly used hydrophilic polymers, drug tends to crystallize out of the initially homogeneous drug/polymer solution during storage, especially at high drug loading and/or when exposed to moisture.

\section{REFERENCES}

[1] Seehra, M. S., Rall, J. D., Liu, J. C., \& Roberts, C. B. (2012). Coreshell model for the magnetic properties of $\mathrm{Pd}$ nanoparticles. Materials Letters, 68, 347-349.

[2] Mino, G., \& Kaizerman, S. (1958). A new method for the preparation of graft copolymers. Polymerization initiated by ceric ion redox systems. Journal of Polymer Science, 31(122), 242-243.

[3] Wingender, J., Neu, T. R., \& Flemming, H. C. (Eds.). (2012). Microbial extracellular polymeric substances: characterization, structure and function. Springer Science \& Business Media.

[4] Hajji, P., David, L., Gerard, J. F., Pascault, J. P., \& Vigier, G. (1999). Synthesis, structure, and morphology of polymer-silica hybrid nanocomposites based on hydroxyethyl methacrylate. Journal of Polymer Science Part B: Polymer Physics, 37(22), 31723187.

[5] Burnside, S. D., \& Giannelis, E. P. (1995). Synthesis and properties of new poly (dimethylsiloxane) nanocomposites. Chemistry of materials, 7(9), 1597-1600. 